\title{
How to introduce virtual microscopy (VM) in routine diagnostic pathology: Constraints, ideas, and solutions
}

\author{
Klaus Kayser ${ }^{\mathrm{a}, *}$, Stephan Borkenfeld ${ }^{\mathrm{b}}$ and Gian Kayser ${ }^{\mathrm{c}}$ \\ ${ }^{\mathrm{a}}$ Charite, University of Berlin, Berlin, Germany \\ ${ }^{\mathrm{b}}$ IAT (International Academy of Pathology), Heidelberg, Germany \\ ${ }^{\mathrm{c}}$ Institute of Pathology, University of Freiburg, Freiburg, Germany
}

\begin{abstract}
Context: Virtual microscopy which is the diagnostic work with digitized microscopic images in tissue - based diagnosis is in its childhood in being implemented in routine diagnosis. Until today, only a few pathology institutions take use of this new technology, although it is available since several years. Why?

Design: Virtual microscopy requires a new workflow organisation in the pathologist's diagnostic procedure. At a first view, the laboratory workflow seems to remain untouched to a high degree. However, the used laboratory information system (LIS), which is commonly built in a hierarchic order, has to be adjusted at its highest levels, i.e., diagnosis statement, quality evaluation, submission to the clinician (hospital information system), and feedback to the laboratory. Therefore, the laboratory's workflow is involved at all levels too, and the LIS has to be changed or adjusted to the requirements of VM. VM systems are usually equipped with a viewer that mimics the viewing of a conventional microscope, and do not offer access to sensitive nodes of the LIS. Similar, LIS are usually closed and fixed systems because of data security and certification demands. Thus, VM systems have to possess communication access at different LIS levels together with steering commands for the LIS in close association with the diagnostic quality and efficiency (for example demands for additional stains, immunohistochemical or quantitative image methods, etc.), as well as expert consultation, or panel discussion.

Outcome: An implementation of an open and active LIS - VM management system could significantly promote the introduction of VM into routine diagnostic surgical pathology. The management system has to coordinate and translate the demands of VM to LIS (and vice versa), and to assure the communication with HIS. Mandatory features include streaming of the laboratory workflow, feedback commands to LIS, as well as regulation of temporary priority levels.

Conclusion: A successful implementation of VM systems in routine tissue-based diagnosis requires communicative management systems as long as VM is considered to be a "stand alone system" that just mimics a conventional microscope.
\end{abstract}

Keywords: Virtual microscopy, pathology laboratory workflow, laboratory information system, EAMUS

\section{Introduction}

Without any doubt, surgical pathology or tissue based diagnosis is awaiting remarkable changes in the next few years. These will include the diagnostic work with completely digitized glass slides (virtual slides), the application of newly developed mark-

${ }^{*}$ Corresponding author: Klaus Kayser, Charite, University of Berlin, Berlin, Germany. E-mail: klaus.kayser@charite.de. ers for demonstration (visualization) of intra-cellular pathways, and an intensive collaboration with those colleagues who treat the patient, i.e., the oncologist and/or surgeon. The keyword of this new world is called individualized (cancer) therapy or predictive diagnosis $[1,2]$. The formal procedure includes a series of steps of tissue examinations in a hierarchical order. It starts with the conventional analysis of small tissue fragments (H\&E stained biopsies), followed by analysis of receptors bound to cellular 
membranes and involved in information/recognition processes of cellular division (and apoptosis). The final step includes the analysis of intra-cellular pathways that transfer the extracellular information to the genetic data base (genes) that is responsible for the release of reduplication/apoptosis signals. The series of mandatory examinations is called predictive diagnosis, as its results steer the treatment of the individual patient in close association with the obtained intra- and extracellular functions. An accurate and reproducible as well as efficient implementation of these procedures require in addition to standardized fixation, tissue processing, conventional and immunohistochemical (IH) staining methods sophisticated molecular biology and genetic techniques, and their monitoring [3-5].

A human based interactive judgement of the obtained IH signals still is in use in most of the involved pathology institutions. These are subject to non negligible inter- and intra-observer variations. The same holds true for other procedures, for example based upon fluorescent signals. Accurate measurements are sensitive to digital measurement protocols, they can, however in contrast to observer variations reproducible analyzed and, if necessary, corrected [6-10].
Therefore, the recently developed molecular biology techniques need digital image acquisition and associated analysis programs [11-15]. This performance, on the other hand, will imply remarkable changes in the daily workflow of a surgical pathology institution [2, 3, 16-18].

In this article we want to describe, analyze and judge the necessary, probably unavoidable changes with their benefits and constraint in a surgical pathology institution. Even at this early stage of our article we want to claim that surgical pathology has to follow the new development, if it wants to maintain tissue based diagnosis in its hands. Otherwise it will loose its competence, and, in addition, non-negligible financial resources.

\section{Workflows in routine diagnostic surgical pathology}

\subsection{Conventional workflow}

The workflow of an institute of surgical pathology plays an important role in performance and poten-

\section{Workflow of a conventional pathology institution}

\section{Hospital Information System (HIS)}

- Acquisition of patient's data

Laboratory information System (LIS)

- Tissue identification - patient

- Sampling of adequate tissue probes

- Tissue preparation $\rightarrow$ glass siride(s)

- Pathologist (Information System)

- Side examination (image analysis)

- Diagnosis report (preliminary/definitive)

- Further analysis (expert consultation)

- Hospiltal Inffiormation System (HIIS)
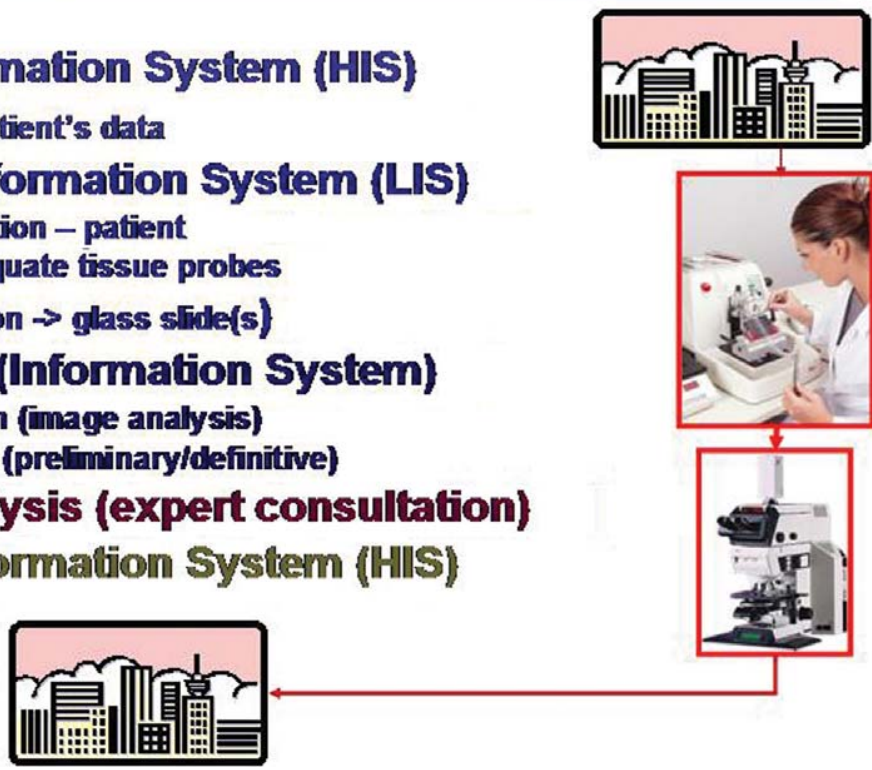

Fig. 1. Workflow of routine diagnostics in a conventional institute of pathology. 
tial changes or introduction of new technologies [3, 18]. Its basic features are displayed in (Fig. 1). The components of the workflow include the Hospital Information System (HIS), the Laboratory Information System (LIS), the Pathologist's Information System (PIS) including the pathologist him/herself, and some additional, not mandatory components such as Expert Consultation System (ECS) or Diagnosis Assurance System (DAS) in larger Institutes of Pathology [2, 19-22]. The HIS assures the fast and correct transfer of the patient's data to the Institute of Pathology, and the biunique connection of the tissue with the patient. It is commonly replaced by a so - called patient card in smaller Institutes of Pathology which handle tissue submitted by private medical practitioners. The LIS assures the biunique data transfer between the tissue and the derived glass slides that are viewed by the pathologist. The obtained findings including the derived diagnosis are usually dictated, documented and transferred to the HIS by a specific Pathology Information System (PIS) that also handles the financial aspects (reimbursement) in general. These components are necessary and usually form the general workflow of an effective and structured Institute of Pathology [1, 10, 23-26]. Additional components include consultation of experts or access to specific cases collections (atlases) in difficult or non familiar cases. The described workflow assures a time effective and "continuous" work of the pathologist who is involved in about 20-60 cases (patients) per day. In addition, VSs are an adequate tool to be applied in intra-operative frozen section diagnosis [27].

How to include virtual microscopy (VM) in a matured and effective workflow?

\subsection{Workflow with included (interactive) Virtual Microscopy (VM)}

At a first glance, the general scheme of such a workflow differs only minimal from the conventional one (Fig. 2). Only two components have to be added between the LIS and the PIS, namely the Virtual Microscopy (VM), and an electronic storage and retrieval system (ESRS), if we want to include the pathologist's role in a system derived from conventional workflow, i.e., with human - based assessment of diagnosis and without machine assistants. However, these two additional components alter the workflow to a large extent:

\section{Workflow of virtual microscopy in a pathology institution}

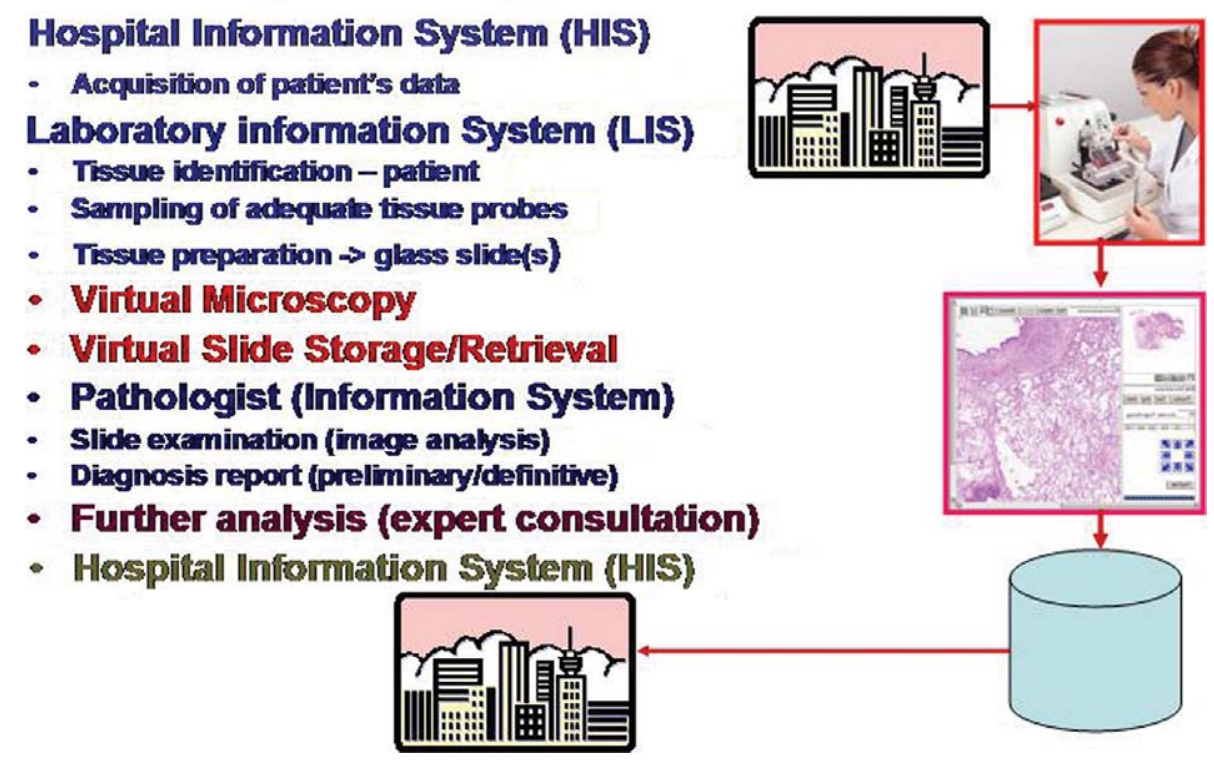

Fig. 2. Workflow of routine diagnostics with included virtual microscopy in an institute of pathology. 


\section{Interactive features}
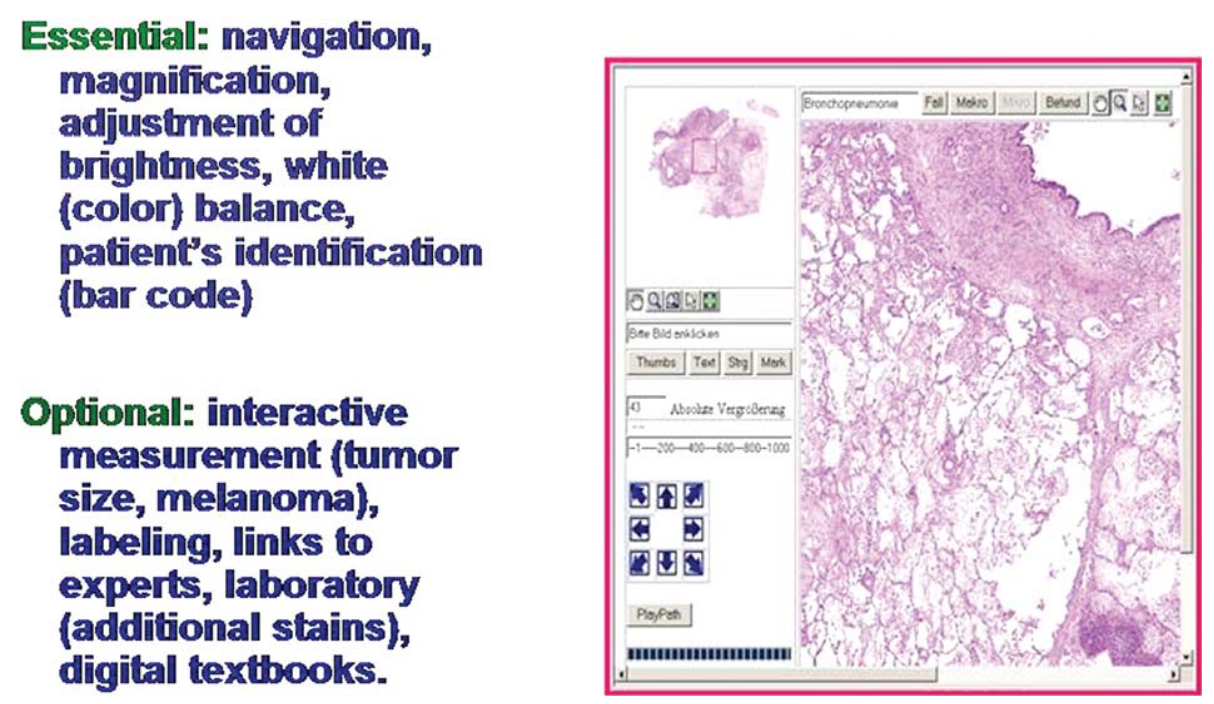

Fig. 3. Essential and optional interactive features in a VM.

VM consists of several components in a time oriented hierarchical order. The first component is the image acquiring system (scanner) that has to be loaded with the conventional glass slides. Most systems require magazines of 20-50 glass slides/magazine [28, 29]. In addition, a bar code is mandatory to assure a correct and automated identification of the obtained virtual slide (VS) with the glass slide. The second component is the fast and error - free transmission of the obtained (usually large, i.e., several GB in size) VSs to an image data bank. The third component steers the presentation of the VSs to the pathologist, i.e., combines VSs of different stains with the patient's data such as clinical history, radiological findings, etc. Some VMs posses components of the PIS such as ordered presentation of the cases, or included digital dictation, others not. In most cases the pathologist has to type in her/his findings and diagnosis. Aspects of reimbursement have not been included in any of the commercially available systems to our knowledge at present.

What are the specific features of such an interactive workflow?

\subsection{Specific features of interactive Virtual Microscopy (VM)}

VM transfers the stained tissue of a conventional glass slide into an electronic image (VS). The aim of the pathologists work remains the same: To derive as much information as possible from the image(s) and transfer this information into the medical language which is the diagnosis. Therefore, the principle work using interactive VM is closely related to the work with a conventional glass slide. Essential are navigation, magnification, illumination, and color adjustment (correction) in addition to the patient's data (Fig. 3). However, the digital presentation of the images permits additional interactive assessments such as measurements of size (melanoma), labelling of specific findings (demonstration of giant cells, etc.), crude frequency analysis (counting of mitoses via a grid), overlay or contemporary display of different stains (tumor classification due to cytoskeleton expression), instructions to the LIS (request for additional stains, etc.).

The implementation of interactive VM implies additional work in the laboratory because of loading and maintenance of the scanner and related issues. This additional workload is compensated by digitized storage and retrieval of VS as well as less workload of the pathologist [1, 10, 19, 30-33]. The main constraint of implementation and use of interactive VM are the lower velocity of image screening (sampling) and sometimes the non-appropriate adjustment of image size on the screen as well as missing individual color adjustment [31, 34-36]. Whether one or two screens are more suitable in interactive VM is 


\section{Interactive Virtual Microscopy: one or two monitors?}

\section{Basics of two monitors display}
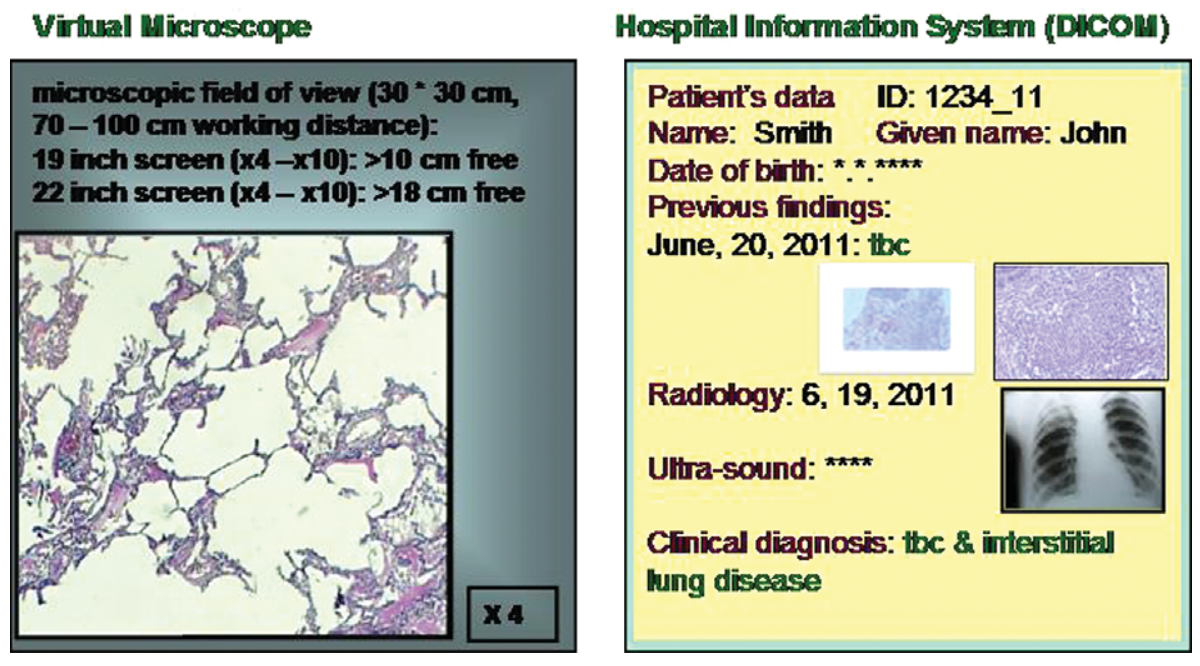

Fig. 4. Basic scheme of information display in a two-monitor VM system.

still under discussion; however, a two screen solution as demonstrated in (Fig. 4) seems to have more advantages.

\subsection{Specific features of automated Virtual Microscopy (VM)}

Automated VM differs from interactive VM in several points: The aim of automated VM is a support of the pathologist's work by so-called electronic assistants (comparable with those used in other service programs such as corel draw, excel, power point, word or similar programs) $[1,10,23]$. None of these assistants is commercially available to our knowledge at present; however, several successful systems have already been reported. They include "a complete program" of programs arranged in a hierarchical order. They start with analysis (and potential adjustment) of image quality, followed by recognition of region of interest (ROI) and the most appropriate magnification for object segmentation and identification $[4,25,30$, $31,37-40]$. The last step is an automated classification of the "image information carriers" which are derived from objects, structures, and textures [1, 31, 36]. The so-called image content information is then compared with quality assurance sets that include the already obtained diagnoses and clinical data such as age, sex, symptoms, etc. and the related probability of the electronically evaluated diagnosis $[1,31,36]$. The pathologist's duty would be to judge distinct steps in the described procedure, to assure and finalize the obtained diagnosis.

The workflow of such a system is shown in (Fig. 5), a demonstration of a potential arrangement at a "supervision screen" is depicted in (Fig. 6). Some programs that are included in these algorithms cannot be judged visually. These include the texture analysis and proposed segmentation algorithms which can vary according to the image quality and automated correction $[1,6,34$, $35,41-45]$.

\section{Perspectives of Virtual Microscopy}

Without any doubt, the development of mandatory VM components has made a remarkable progress in the last few years. Commercially available virtual slide scanners have been released that acquire a whole glass slide image in less than $60 \mathrm{sec}$ with a pixel resolution $<0.2 * 0.2 \mu \mathrm{m}^{2}$. Fast transfer of the several GB measuring images in combination with advanced 


\section{Workflow of advanced virtual microscopy in a pathology institution}

Hospital Information System (HIS)

- Acquisition of pativent's data

Laboratory information System (LIS)

- Tissue identification - patient

- Sampling of adequate tissue probes

- Tissue preparation $\rightarrow$ glass slide(s)

- Virtual Microscopy \& Assistants

- Virtual Slide Storage/Retrieval

- Pathologists Diagnosis System

- Slide examination (image analysis)

- Diagnosis report (preliminary/definitive)

- Supervision (Pathologist's consultation system)

- Hospiltall Infirommation System (HIIS)

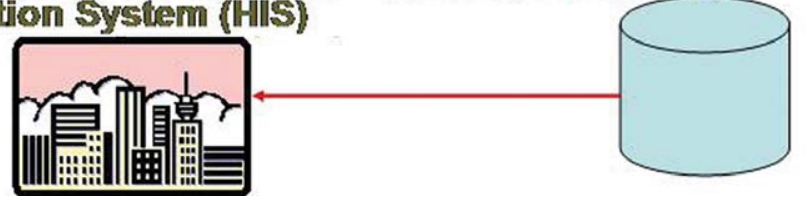

Fig. 5. Workflow of routine diagnostics in an institute of pathology equipped with an advanced VM system.

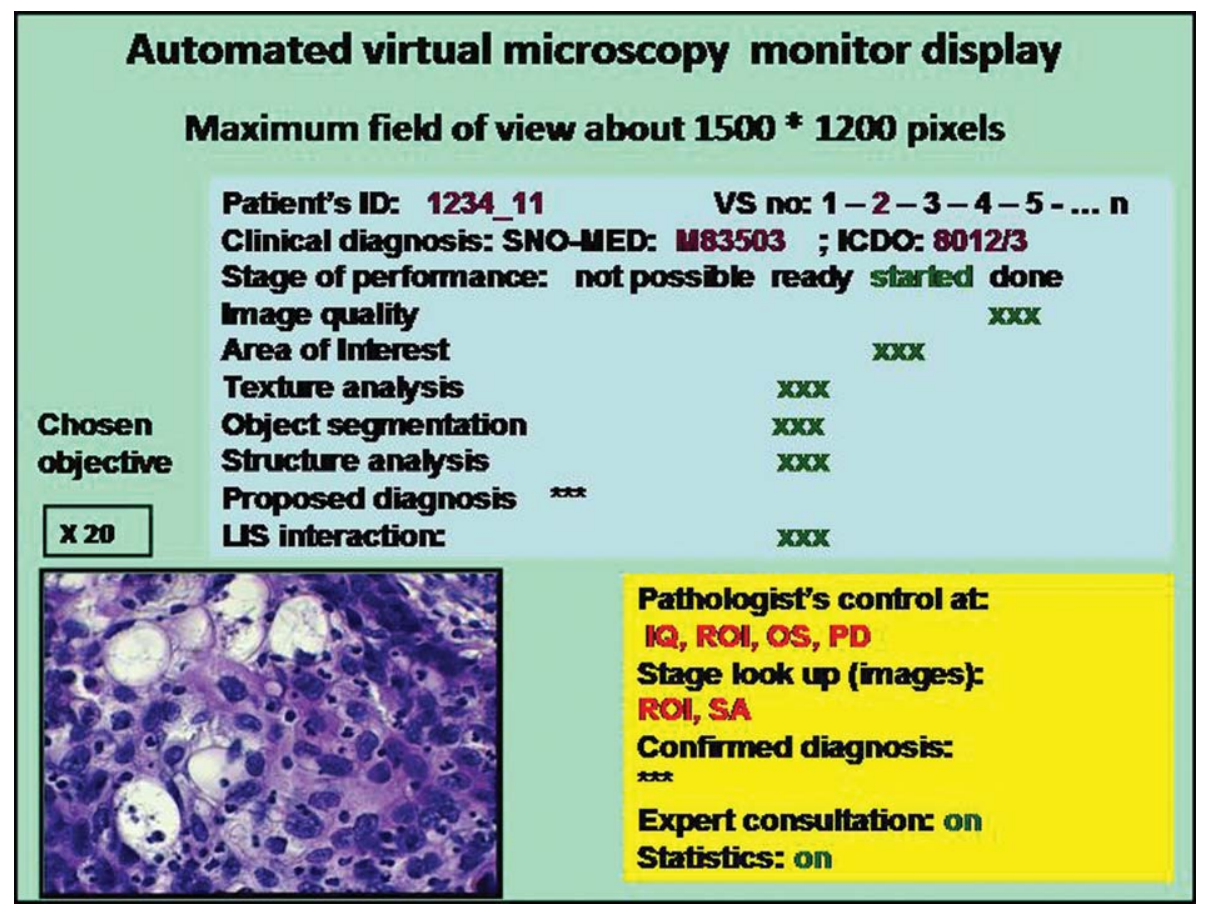

Fig. 6. Proposed scheme of monitoring an automated VM by the pathologist. 
image compression algorithms is available too [17, 22, 46-49]. Some companies have optimized the related image viewers to the latest communication standards and technology such as FLASH, HTML5/AJAX, (hypertext markup language), asynchronous Java script and XML (extensible mark up language), Silver light, etc. Sophisticated image analysis tools are also available $[6,9,21,23,35,43,50,51]$. They mainly focus on immunohistochemistry and "translate" the measured absorption values of the applied visualization dye (DAB, AP, etc.) to the visual judgement of a pathologist (scores) $[12,35,36,52,53]$. Some of these systems have already been approved by the US Food and Drug Administration (FDA).

Until today, numerous systems have been sold, and installed in larger pathology institutions to our knowledge. What are they mainly used for?

They all possess components that permit a reliable image acquisition and transfer, a useful tool for teaching and interdisciplinary case demonstration. Nearly all pathology institutes teach students, junior assistants and provide clinical - pathology conferences using virtual slides [54-58]. These issues are the main applications of VM. Its implementation into routine diagnostics remains a slow and hesitating process, and only two European Pathology Institutions (in Sweden and in The Netherlands, both private) have released some information about the experiences. Both of them have implemented a specific surface with the LIS, and both of them are still using conventional microscopy contemporary to our knowledge.

However, the maturation of VM in terms of offering flexible and fast surfaces to different LIS and PIS on the basis of HL7 (Health Level 7), DICOM (Digital Imaging and Communication), and PACS (Picture Archiving and Communication Standard) solutions (standardization) has remarkably increased the interest of larger Institutes of Pathology to implement VM and replace the existing conventional systems [34, 59, 60]. Therefore, we expect that at least in the Western European Countries VM systems that focus on routine diagnostics in surgical pathology (tissue - based diagnosis) will be implemented and matured by corresponding practical experiences in the near future. Such systems will slowly turn from interactive to automated use in order to provide the required cost/efficiency balance which can only be obtained if the otherwise sharply increasing workload of the pathologists will be reduced by this modern technology.

\section{References}

[1] K. Kayser, B. Molnar and R. Weinstein, Virtual Microscopy: Fundamentals, Applications, Perspectives of Electronic Tissue-based Diagnosis, VSV Interdisciplinary Medical Publishing, Berlin, 2006, 206.

[2] A.R. Jara-Lazaro, et al., Digital pathology: Exploring its applications in diagnostic surgical pathology practice), Pathology 42(6) (2010), 512-518.

[3] K. Kayser, et al., New developments in digital pathology: From telepathology to virtual pathology laboratory, Stud Health Technol Inform 105 (2004), p. 61-69.

[4] K. Kayser and G. Kayser, Virtual Microscopy and Automated Diagnosis in Virtual Microscopy and Virtual Slides in Teaching, Diagnosis and Research, J. Gu and R. Ogilvie, Editors, Boca Raton, Taylor Francis, 2005.

[5] P. Bertheau, H. Kussaibi and D. Ameisen, [Virtual slides: Technical aspects and an example of their use at a university hospital], Ann Pathol 28, Spec No 1(1) (2008), p. S98-S99.

[6] K. Kayser, et al., How to measure diagnosis-associated information in virtual slides, Diagn Pathol 6(Suppl 1) p. S9.

[7] R.K. Kumar, et al., Virtual microscopy for learning and assessment in pathology, J Pathol 204(5) (2004), 613-618.

[8] J. Stewart, 3rd, et al., Virtual microscopy for cytology proficiency testing: Are we there yet? Cancer 111(4) (2007), 203-209.

[9] K. Kayser, et al., Texture- and object-related automated information analysis in histological still images of various organs, Anal Quant Cytol Histol 30(6) (2008), 323-335.

[10] K. Kayser, et al., Digitized pathology: Theory and experiences in automated tissue-based virtual diagnosis, Rom J Morphol Embryol 47(1) (2006), 21-28.

[11] F. Curreli, et al., Virtual screening based identification of novel small-molecule inhibitors targeted to the HIV-1 capsid, Bioorg Med Chem 19(1) (2011), p. 77-90.

[12] J. Konsti, et al., Development and evaluation of a virtual microscopy application for automated assessment of Ki-67 expression in breast cancer, BMC Clin Pathol 11 (2011), 3.

[13] M.J. Machado, et al., Dynamics of angiogenesis during wound healing: A coupled in vivo and in silico study, Microcirculation 18(3) (2011), 183-197.

[14] C.G. Bologa, et al., Virtual and biomolecular screening converge on a selective agonist for GPR30, Nat Chem Biol 2(4) (2006), 207-212.

[15] R. Kiesslich, M. Goetz and M.F. Neurath, Virtual histology, Best Pract Res Clin Gastroenterol 22(5) (2008), 883-897.

[16] L. Roa-Pena, F. Gomez and E. Romero, An experimental study of pathologist's navigation patterns in virtual microscopy, Diagn Pathol 5 (2010), 71.

[17] D. Romo, E. Romero and F. Gonzelez, Learning regions of interest from low level maps in virtual microscopy, Diagn Pathol 6(suppl 1) (2011), S22.

[18] T. Kalinski, et al., Digital workflow management for quality assessment in pathology, Pathol Res Pract 204(1) (2008), 17-21.

[19] P.S. Nielsen, et al., Virtual microscopy: An evaluation of its validity and diagnostic performance in routine histologic diagnosis of skin tumors, Hum Pathol 41(12) (2010), 17701776 . 
[20] R.S. Weinstein, et al., Telepathology overview: From concept to implementation, Hum Pathol 32(12) (2001), 1283-1299.

[21] F. Demichelis, et al., The virtual case: A new method to completely digitize cytological and histological slides, Virchows Arch 441(2) (2002), 159-164.

[22] S. Wienert, et al., Integration and acceleration of virtual microscopy as the key to successful implementation into the routine diagnostic process, Diagn Pathol 4 (2009), 3.

[23] K. Kayser, et al., Interactive and automated application of virtual microscopy, Diagn Pathol 6(Suppl 1) (2011), S10.

[24] L. Pantanowitz, et al., Review of the current state of whole slide imaging in pathology, J Pathol Inform 2 (2011), 36.

[25] K. Kayser, et al., AI (artificial interlligence): From Image Analysis via Automated Diagnosis to Grid Technology, Journal of Health Technology and Application 8(3) (2010), 88-93.

[26] K. Kayser, et al., Grid computing in image analysis, Diagn Pathol 6(Suppl 1) (2011), S12.

[27] Y. Tsuchihashi, et al., Use of virtual slide system for quick frozen intra-operative telepathology diagnosis in Kyoto Japan, Diagn Pathol 3(Suppl 1) (2008), S6.

[28] L. Ficsor, et al., Automated virtual microscopy of gastric biopsies, Cytometry B Clin Cytom 70(6) (2006), 423-431.

[29] B. Molnar, et al., Digital slide and virtual microscopy based routine and telepathology evaluation of routine gastrointestinal biopsy specimens, J Clin Pathol 56(6) (2003), 433-438.

[30] P. Belhomme, et al., Towards a computer aided diagnosis system dedicated to virtual microscopy based on stereology sampling and diffusion maps, Diagn Pathol 6(Suppl 1) (2011), S3.

[31] K. Kayser, et al., Towards an automated virtual slide screening: Theoretical considerations and practical experiences of automated tissue-based virtual diagnosis to be implemented in the Internet, Diagn Pathol 1 (2006), 10.

[32] S.H. Lee, Virtual microscopy: Applications to hematology, Lab Hematol 11(1) (2005), 38-45.

[33] M. Lundin, J. Lundin and J. Isola, Virtual microscopy, J Clin Pathol 57(12) (2004), 1250-1251.

[34] K. Kayser, et al., Image standards in tissue-based diagnosis (diagnostic surgical pathology), Diagn Pathol 3 (2008), 17.

[35] K. Kayser, et al., How to measure image quality in tissuebased diagnosis (diagnostic surgical pathology), Diagn Pathol 3(Suppl 1) (2008), S11.

[36] K. Kayser, et al., Theory of sampling and its application in tissue based diagnosis, Diagn Pathol 4 (2009), 6.

[37] R. Ferreira, et al., The Virtual Microscope, Proc AMIA Annu Fall Symp (1997), 449-453.

[38] K. Kayser, Quantification of virtual slides: Approaches to analysis of content-based image information, J Pathol Inform 2 (2011), 2.

[39] A.P. King, et al., A system for microscope-assisted guided interventions, Stereotact Funct Neurosurg 72(2-4) (1999), $107-111$.

[40] R.S. Weinstein, et al., Overview of telepathology, virtual microscopy, and whole slide imaging: Prospects for the future, Hum Pathol 40(8) (2009), 1057-1069.

[41] K. Glatz-Krieger, et al., Factors to keep in mind when introducing virtual microscopy, Virchows Arch 448(3) (2006), 248-255.
[42] H. Gongora Jara and H.A. Bercelo, Telepathology and continuous education: Important tools for pathologists of developing countries, Diagn Pathol 3(Suppl 1) (2008), S24.

[43] J. Gortler, et al., Grid technology in tissue-based diagnosis: Fundamentals and potential developments, Diagn Pathol 1 (2006), 23.

[44] P. Hufnagl and K. Schluns, [Virtual microscopy and routine diagnostics. A discussion paper], Pathologe 29(Suppl 2) (2008), 250-254

[45] K. Kayser, G. Kayser and K. Metze, The concept of structural entropy in tissue-based diagnosis, Anal Quant Cytol Histol 29(5) (2007), 296-308.

[46] J.P. Johnson, et al., Using a visual discrimination model for the detection of compression artifacts in virtual pathology images, IEEE Trans Med Imaging 30(2) (2011), 306-314.

[47] J.S. Strauss, et al., Virtual microscopy and public-key cryptography for Internet telepathology, J Telemed Telecare 5(2) (1999), 105-110.

[48] T. Kalinski, et al., Lossy compression in diagnostic virtual 3dimensional microscopy-where is the limit? Hum Pathol 40(7) (2009), 998-1005.

[49] V.J. Tuominen and J. Isola, The application of JPEG2000 in virtual microscopy, J Digit Imaging 22(3) (2009), 250-258.

[50] V. Della Mea, 25 years of telepathology research: A bibliometric analysis, Diagn Pathol 6(Suppl 1) (2011), S26.

[51] A. Evered and N. Dudding, Accuracy and perceptions of virtual microscopy compared with glass slide microscopy in cervical cytology, Cytopathology, 22(2) (2011), 82-87.

[52] H.O. Helin, et al., Virtual microscopy in prostate histopathology: Simultaneous viewing of biopsies stained sequentially with hematoxylin and eosin, and alpha-methylacyl-coenzyme A racemase/p63 immunohistochemistry, J Urol 175(2) (2006), 495-499.

[53] A.M. Lopez, et al., Virtual slide telepathology enables an innovative telehealth rapid breast care clinic, Hum Pathol 40(8) (2009), 1082-1091.

[54] G. Campbell, L.A. Demetriou and T.R. Arnett, Virtual histology in the classroom and beyond, Med Educ 44(11) (2010), $1124-1125$.

[55] L. Helle, et al., Traditional microscopy instruction versus process-oriented virtual microscopy instruction: A naturalistic experiment with control group, Diagn Pathol 6(Suppl 1) (2011), S8.

[56] K. Kayser, et al., E-education in pathology including certification of e-institutions, Diagn Pathol 6(Suppl 1) (2011), S11.

[57] S. Raja, Virtual microscopy as a teaching tool adjuvant to traditional microscopy, Med Educ 44(11) (2010), 1126.

[58] J. Szymas and M. Lundin, Five years of experience teaching pathology to dental students using the WebMicroscope, Diagn Pathol 6(Suppl 1) (2011), S13.

[59] R. Zwonitzer, et al., Digital pathology: DICOM-conform draft, testbed, and first results, Comput Methods Programs Biomed 87(3) (2007), 181-188.

[60] M. Garcia Rojo, et al., Digital pathology in Europe: Coordinating patient care and research efforts, Stud Health Technol Inform 150 (2009), 997-1001. 


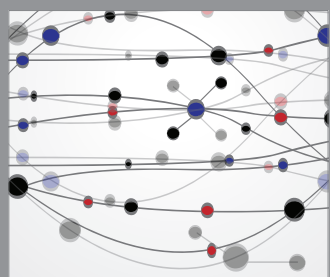

The Scientific World Journal
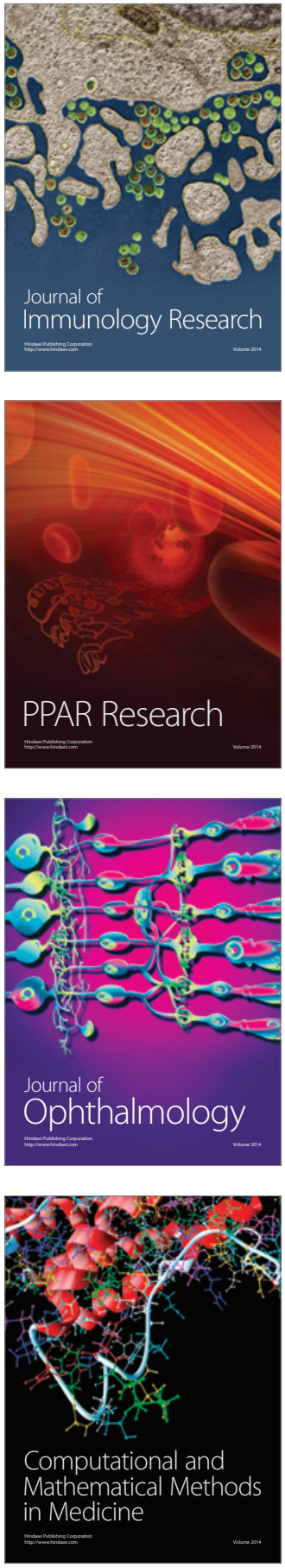

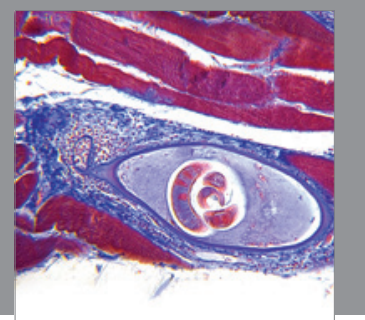

Gastroenterology

Research and Practice
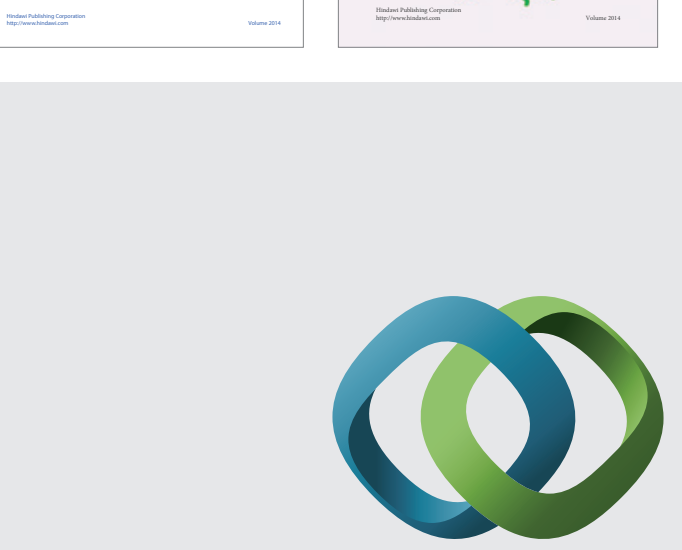

\section{Hindawi}

Submit your manuscripts at

http://www.hindawi.com
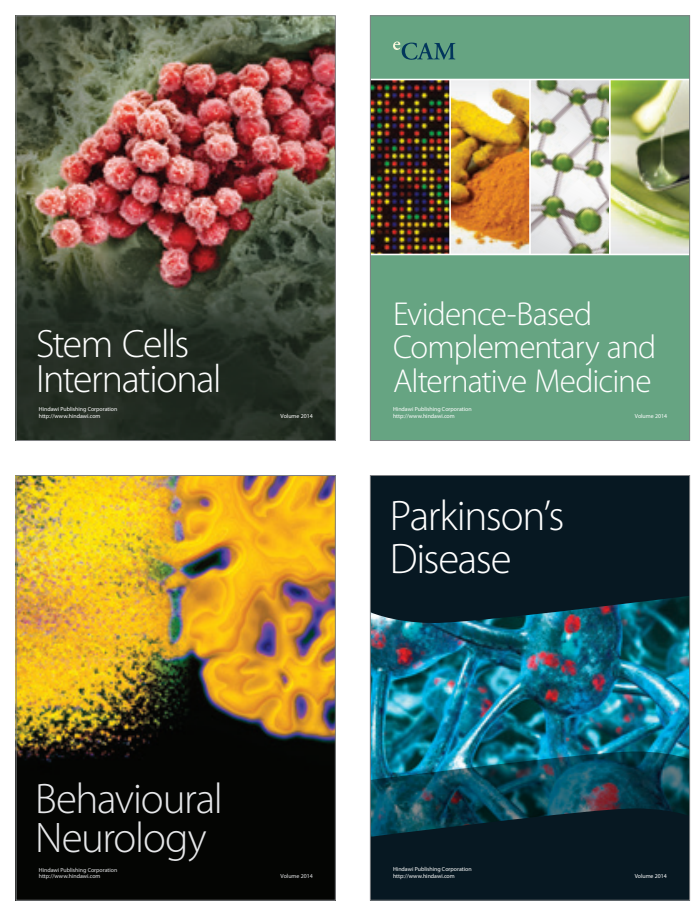

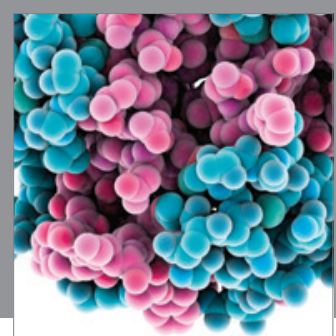

Journal of
Diabetes Research

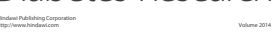

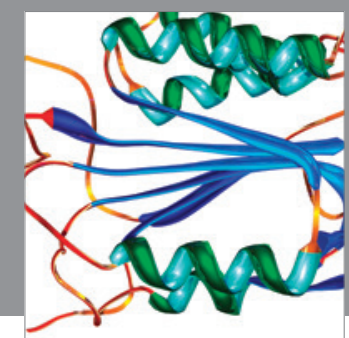

Disease Markers
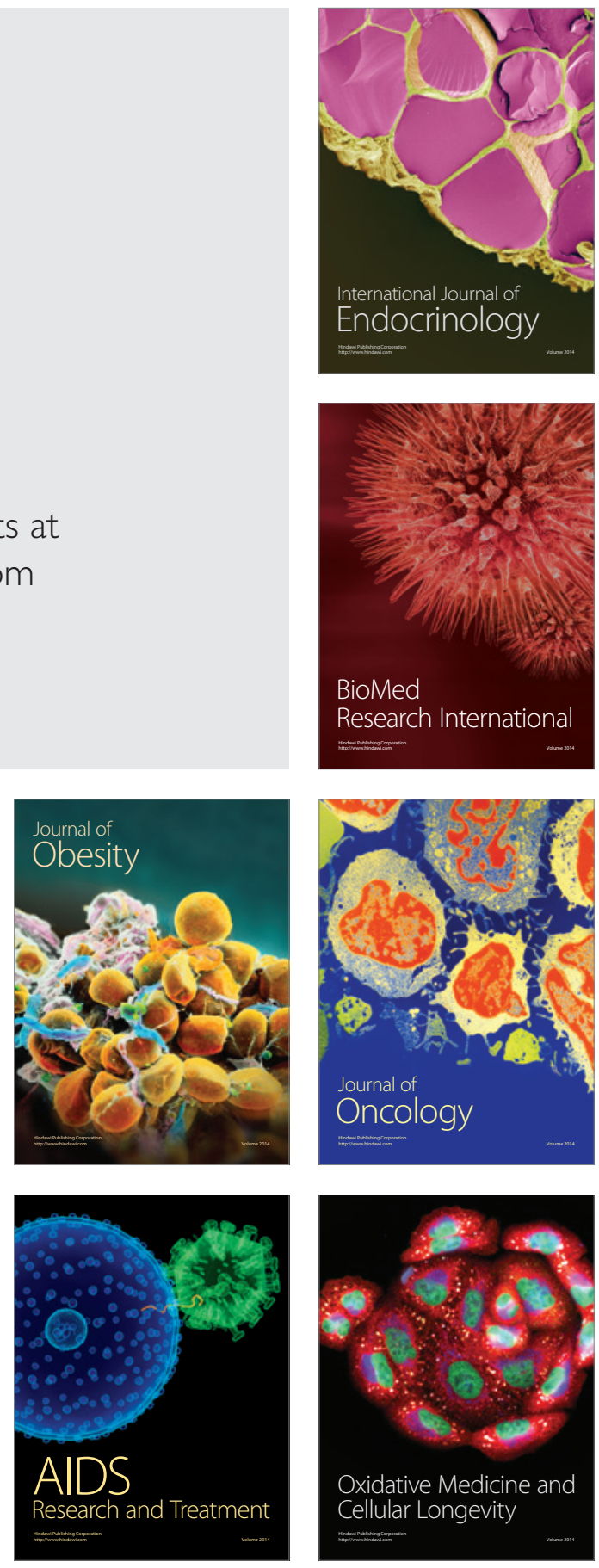